\title{
Synthesis and characterization of bioactive hydroxyapatite/ fluoroapatite solid solutions using corals ${ }^{\dagger}$
}

\author{
T S SAMPATH KUMAR, M SIVAKUMAR, N PRASANTH KUMAR, \\ K SENTHAMIL SELVI* and K PANDURANGA RAO* \\ Department of Nuclear Physics and Materials Science Centre, University of Madras, Madras \\ 600025. India \\ *Biomaterials Division, Central Leather Research Institute, Madras 600020, India
}

\begin{abstract}
Hydroxyapatite/fluoroapatite solid solutions were prepared by two different methods using Indian coast corals. In the 'low temperature method' a known volume $(50 \%)$ of hydrofluoric acid was added to the coral and an exchange reaction with diammonium phosphate was carried out to form apatite at $250^{\circ} \mathrm{C}$ under hydrothermal conditions. The coralline derived hydroxyapatite by the above method was reacted with 50 mol\% sodium fluoride at $900^{\circ} \mathrm{C}$ in the 'high temperature method'. The X-ray powder diffraction, infrared spectroscopy and thermal analysis techniques were employed to characterize the fluorination of the hydroxyapatite. In vitro solubility study in phosphate buffer solution of $\mathrm{pH} 7.2$ indicate the stability of the apatites. The low temperature method of fluorination seems to be advantageous due to better in vitro stability and absence of impurity phases.
\end{abstract}

Keywords. Hydroxyapatite; fluoroapatite; corals; bone substitutes.

\section{Introduction}

Hydroxyapatite (also known as hydroxylapatite, HAp) is a potential bone substitute material in orthopedics and dentistry due to its excellent biocompatible and osteoconductive properties. The osteoconductive behaviour of HAp imply that, when implanted in skeletal tissue, new bone formation is guided along the HAp surface, promoting bone growth into areas that it otherwise would not occupy (Damien and Parsons 1991). However, the mechanical strength of HAp is fairly poor and therefore it is not used as bulk implants for load bearing applications such as total hip replacement.

Attempts were made to improve the mechanical strength by substitution of certain ions like fluorine in HAp (Hench 1991). Apatite forms solid solutions with fluoroapatite (Roth and Negas 1983). Although the lattice parameters are approximately the same, $\left[\mathrm{Ca}_{10}\left(\mathrm{PO}_{4}\right)_{6}(\mathrm{OH})_{2}\right.$, ASTM card no. 9-432, $a_{0}=0.9418 \mathrm{~nm}, c_{0}=0.6884 ; \mathrm{Ca}_{10}\left(\mathrm{PO}_{4}\right)_{6} \mathrm{~F}_{2}$, ASTM card no. 15 -876, $\left.a_{0}=0.9364, c_{0}=0.6884\right]$, the $a$-axis of the HAp crystal decreases with the increasing degree of fluorination (Moreno et al 1977). The fluorine substituted HAp possesses a notably higher corrosion resistance in biological environment (Narasaraju 1972). Stoichiometric fluoroapatite possesses a fluorine content of $3.77 \mathrm{wt} \%$ while the fluorine content of the human bone is around $1 \mathrm{wt} \%$ (Osborn and Newesely 1980). Hence, partially fluorinated HAp can be prepared which is even less soluble than either hydroxyapatite and fluoroapatite under similar conditions (Okazaki 1992; Partenfelder et al 1993).

The preparation of HAp ranges from conventional wet synthesis, solid state reaction method to hydrothermal exchange process in which the calcium carbonate skeleton of a marine coral is converted into HAp. The resultant material is called coralline HAp

${ }^{\dagger}$ Paper presented at the poster session of MRSI AGM VI. Kharagpur, 1995 
(Roy and Linnehan 1974). As India has a vast resource of corals, the development of bone substitutes using these corals have been investigated. In the present study the preparation of coralline derived hydroxyapatite/fluoroapatite solid solutions have been described. Incorporation of the fluorine in the apatite lattice was examined by X-ray diffraction, infrared spectroscopy. thermal analysis methods and by solubility study in phosphate buffer. The results were compared with those for coralline hydroxyapatite.

\section{Experimental}

\subsection{Preparation}

The coral genus 'Goniopora' was obtained from the Gulf of Manner in the southern coast of India. This coral exhibits a porous structure with all pores interconnected throughout the skeleton and the structure resembles that of trabecular bone. The calcareous biomaterial that constitutes coral skeleton consists of $98-99 \%$ calcium carbonate and the remaining part being made up of various amino acids and oligo elements. After gathering, the coral blocks were dried and cleaned of macroscopic impurities. The coral was then heated at $900^{\circ} \mathrm{C}$ for $2 \mathrm{~h}$ to remove the organic materials and impurities. The pre-heated coral is once again washed and dried. The heating makes the coral very brittle which easily becomes powder even under slightest pressure.

Stoichiometric amounts of coral powder, high purity diammonium phosphate and water corresponding to HAp were ground well in a agate mortar and heated in a simplified pressure vessel for few hours at $250^{\circ} \mathrm{C}$ under high pressures of water vapour, for the formation of pure coralline hydroxyapatite (CHAp). Further details of the process are not presented due to economic reasons.

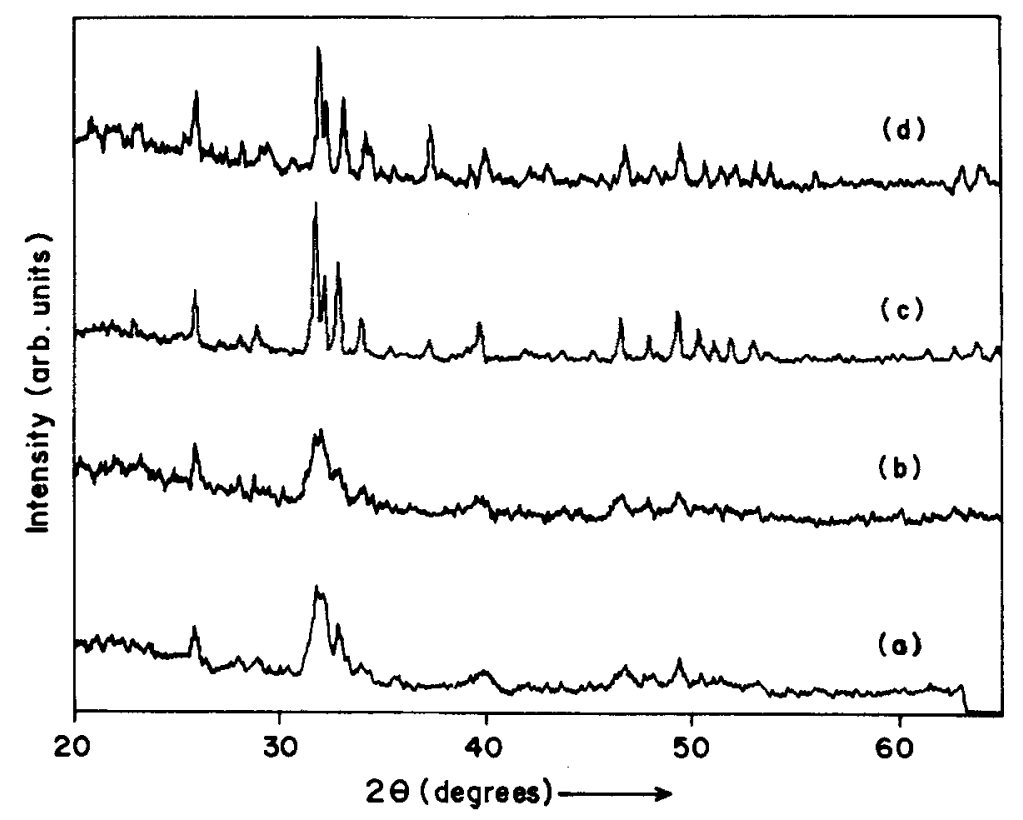

Figure 1. XRD patterns of apatites: (a) CHAp, (b) FHAp(LT), (c) CHAp heated to $900^{\circ} \mathrm{C}$ and (d) FHAp(HT). 
In the 'low temperature method' (LT) of fluorination of CHAp, known volumes of reagent grade hydrofluoric acid was used instead of water in the exchange reaction for the partial fluorination of CHAp. In the 'high temperature method' (HT), the coralline derived HAp obtained by the exchange reaction method is reacted with various amounts of high purity $\mathrm{NaF}$ at $900^{\circ} \mathrm{C}$ for $12 \mathrm{~h}$ in a tubular furnace. The product was washed thoroughly and dried overnight.

\subsection{Characterization}

The powder from the hydroxyapatite/fluoroapatite samples were examined with a Seifert high resolution $\mathrm{X}$-ray powder diffractometer using monochromatic $\mathrm{CuK}_{\alpha}$ radiation in Guinier geometry. The X-ray diffraction (XRD) patterns were recorded in steps of 0.01 degree intervals with one second counting time at each step. Fourier transformed infrared spectroscopy (FTIR) measurements were carried out by the $\mathrm{KBr}$ pellet method using a Nicolet 20 DXB model spectrometer. Thermogravimetry analysis (TGA) and differential thermal analysis (DTA) were performed with PerkinElmer 7 system. The in vitro stability test of the apatites was performed at $37^{\circ} \mathrm{C}$ in buffered phosphate solution of $\mathrm{pH} 7 \cdot 2$ using an incubator and a ECIL $\mathrm{pH}$ meter.

\section{Results and discussion}

The X-ray diffraction patterns of the partially fluorinated coralline hydroxyapatites obtained by both the low temperature method [using $50 \mathrm{vol} \% \mathrm{HF}$, sample code FHAp(LT)] and high temperature method [using $50 \mathrm{~mol} \% \mathrm{NaF}$, sample code FHAp(HT)] are shown in figure 1. The XRD patterns of pure coralline hydroxyapatite (CHAp) and $\mathrm{CHAp}$ heated to $900^{\circ} \mathrm{C}$ are also shown in the same figure for comparison. All the diffractograms show identical patterns. The $900^{\circ} \mathrm{C}$ pre-heated $\mathrm{CHAp}$ and FHAp(HT) exhibited sharp diffraction peaks due to better crystallization at high

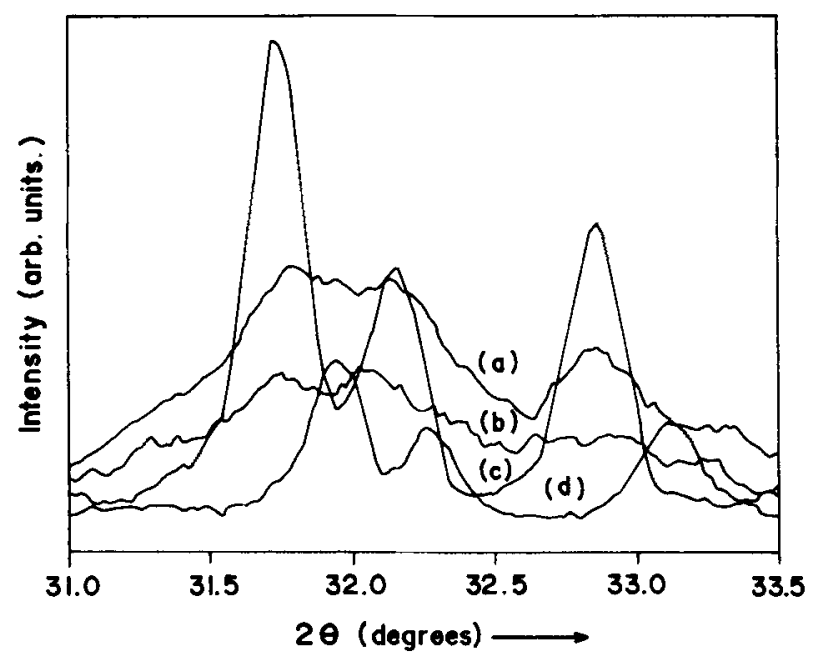

Figure 2. Expanded XRD patterns of apatites in the range of $31^{\circ}$ to $33 \cdot 5^{\circ}$ : (a) CHAp, (b) FHAp(LT), (c) CHAp heated to $900^{\circ} \mathrm{C}$ and (d) FHAp(HT). 
Table 1. List of unit cell parameters.

\begin{tabular}{lcc}
\hline Sample & $a(\AA)$ & $c(\AA)$ \\
\hline HA(ASTM card no. 9-432) & $9 \cdot 42$ & $6 \cdot 88$ \\
FHA(ASTM card no. 15-876) & $9 \cdot 36$ & $6 \cdot 88$ \\
CHA(LT) & $9 \cdot 42$ & $6 \cdot 90$ \\
FHA(LT) & $9 \cdot 38$ & $6 \cdot 90$ \\
FHA(HT) & $9 \cdot 38$ & $6 \cdot 88$ \\
\hline
\end{tabular}

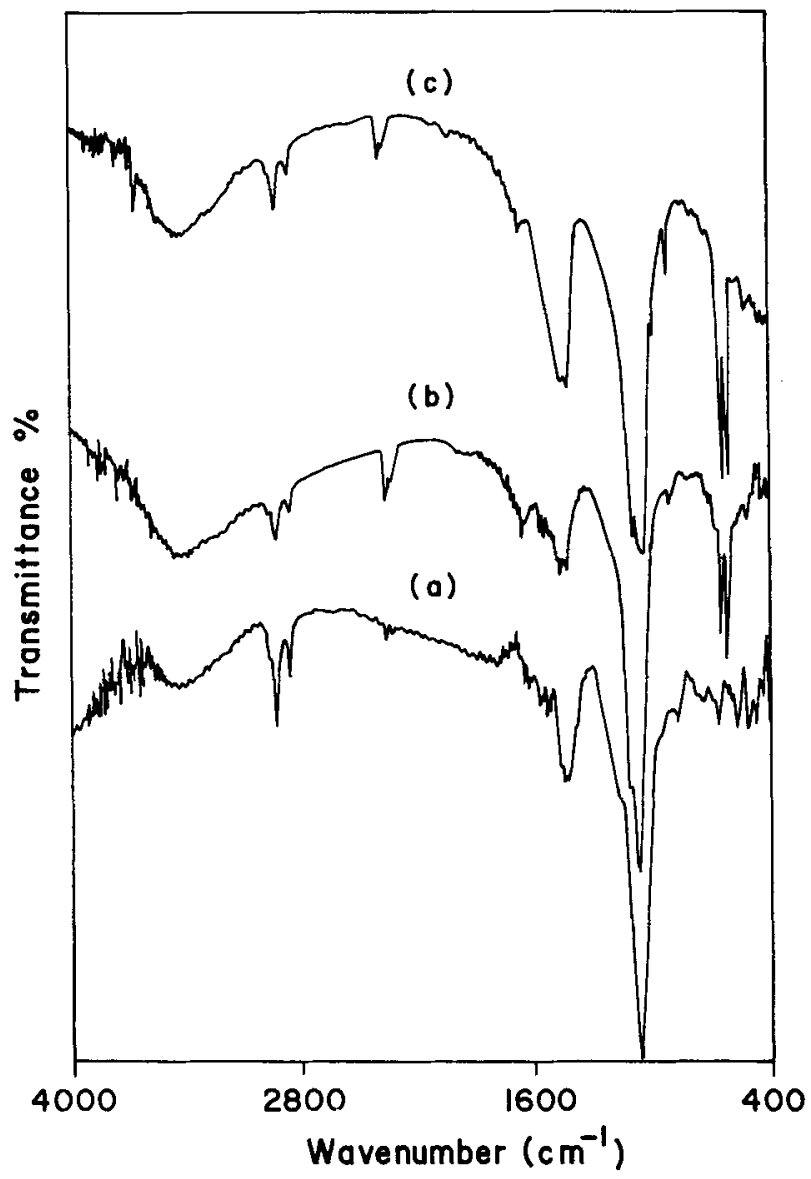

Figure 3. FTIR spectra of apatites: (a) CHAp, (b) FHAp(LT) and (c) FHAp(HT).

temperature. Since, it is well known that the a-axis dimension of the fluorinated hydroxyapatite decreases with the increasing degree of fluorination, the shift of major reflections may be examined as a representation of fluorination (Okazaki 1992; Partenfelder et al 1993). The expanded peaks of the samples in the region of $31.5^{\circ}$ to $33.5^{\circ}$ as shown in figure 2 suggest the fluorination of CHAp especially for the FHAp $(\mathrm{HT})$ sample. The peaks can be indexed using a hexagonal unit cell and the unit cell parameters were obtained by refining its diffraction lines using the standard least square method (CELN) programme. The list of cell parameters are given in table 1 . The 


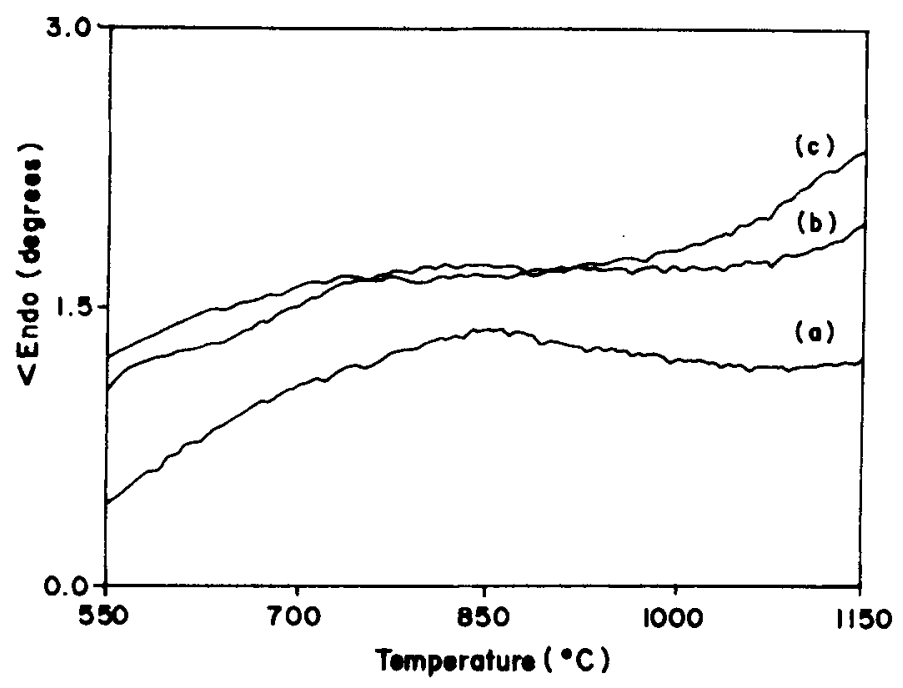

Figure 4. DTA trace of apatites: (a) CHAp, (b) FHAp(LT) and (c) FHAp(HT).

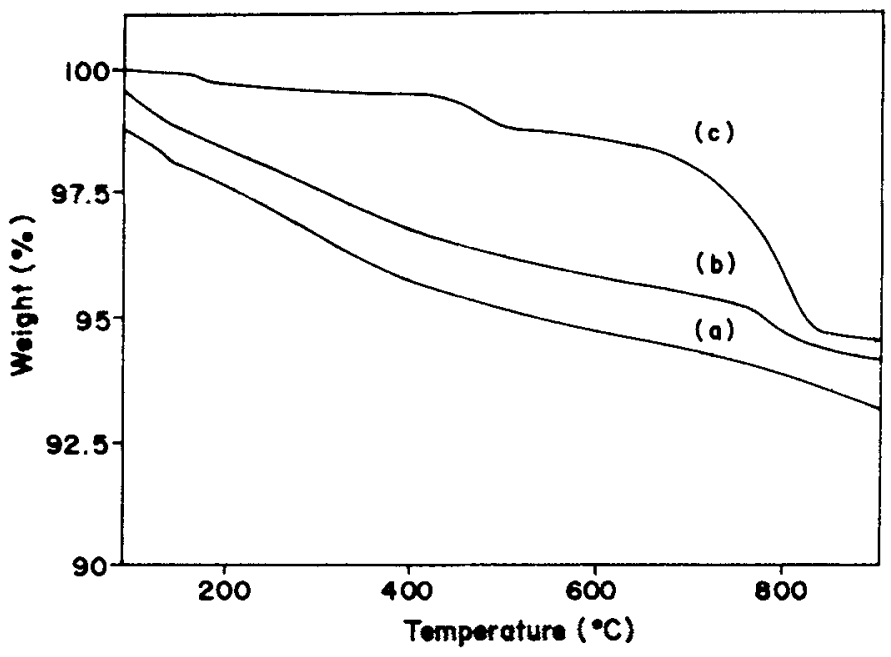

Figure 5. TGA trace of apatites: (a) CHAp, (b) FHAp(LT) and (c) FHAp(HT).

observed reduction of the a-axis parameter for FHAp(LT) and FHAp(HT) samples clearly indicates only partial fluorination (around 60\%) of CHAp.

The FTIR spectroscopy further details the fluorination of CHAp. Figure 3 shows the IR spectra of CHAp, FHAp(LT), and FHAp(HT). The IR spectra of all the samples show absorption bands at $1100 \mathrm{~cm}^{-1}$ and $900 \mathrm{~cm}^{-1}$ due to the $\mathrm{PO}_{4}^{-3}$ ion vibration. The broad absorption at 3000 to $3700 \mathrm{~cm}^{-1}$ in all the samples is due to the $V_{\mathrm{O}-\mathrm{H}}$ vibration. The sharp lines around $600 \mathrm{~cm}^{-1}$ is also due to the vibration of $\mathrm{OH}$ group and shows modification in FHAp due to the fluorination (Okazaki 1992; Partenfelder et al 1993). However, the presence of $\mathrm{CO}_{3}^{-2}$ which appears as a composite of peaks around $1510-1420$ and $800 \mathrm{~cm}^{-1}$ indicated trace contamination by carbonate ions. The FTIR study gave additional proof for fluorination of CHAp. 


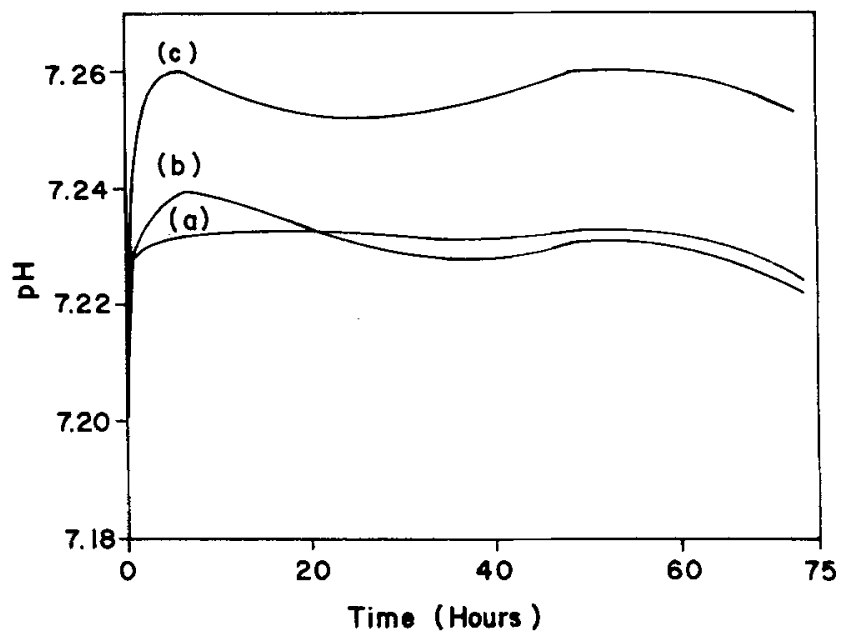

Figure 6. The variations in $\mathrm{pH}$ of phosphate solution at $37^{\circ} \mathrm{C}$ with apatites: (a) $\mathrm{CHAp}$, (b) FHAp(LT) and (c) FHAp(HT).

Thermogravimetric analysis (TGA) and differential thermal analysis (DTA) have also been employed to further characterize the fluoroapatite. The thermograms of TGA and DTA are shown in figures 4 and 5 respectively. As HAp is known to be stable up to $1470^{\circ} \mathrm{C}$ (Locardi et al 1993), the changes observed by DTA between 600 and $1200^{\circ} \mathrm{C}$ may be due to variations of the thermal conductivity of the samples except for the FHAp(HT). Since, this sample was prepared for the high temperature method using $\mathrm{NaF}$, the observed endo peak around $950^{\circ} \mathrm{C}$ may be due to the melting of un-reacted $\mathrm{NaF}$ which may be present in minor quantities. Similarly there is no significant weight loss in the thermogravimetric traces of the samples and the small weight loss observed may be due to the dehydration of apatite according to

$$
\mathrm{Ca}_{10}\left(\mathrm{PO}_{4}\right)_{6}(\mathrm{OH})_{2} \rightarrow \mathrm{Ca}_{10}\left(\mathrm{PO}_{4}\right)_{6}(\mathrm{OH})_{2-2 x}+x\left(\mathrm{H}_{2} \mathrm{O}\right)
$$

However, the weight loss occurs differentially depending upon the method of preparation. Samples prepared by the low temperature method showed continuous weight loss from $90^{\circ} \mathrm{C}$ onwards, while two-step desorption behaviour was observed for samples prepared by the high temperature method. Even though fluorination of CHAp shifts the weight loss to higher temperatures relatively higher weight loss occurred for FHAp(HT) sample as compared to FHAp(LT) sample.

In vitro study of the stability of the fluorinated CHAp and the composites were performed in phosphate buffer solution with $\mathrm{pH} \mathrm{7.2.} \mathrm{The} \mathrm{pH}$ stability of the buffered solutions containing CHAp, FHAp(LT) and FHAp (HT) samples are shown in figure 6. All the samples were found to be stable in the phosphate buffer solution for the period of study. Although fluoroapatite is expected to be less soluble than HAp, the minute variations in the stability observed for the fluorinated CHAp do not exhibit this behaviour. The solubility depends much on the buffering conditions owing to the complex formation (Klein et al 1990). Hence, these results should be observed carefully keeping in view the presence of impurities especially in the FHAp(HT) sample. 


\section{Conclusions}

The present study demonstrated the possibility of fluorination of the HAp derived from corals available in the Indian coast by two different methods. The low temperature method relatively seems to be advantageous due to better in vitro stability and the absence of impurities.

\section{References}

Damien C J and Parsons J R 1991 J. Appl. Biomater. 2187

Hench L L 1991 J. Am. Ceram. Soc. 741487

Klein P A T C, de Blick-Hogervorst J M A. Walke J G C and de Groot K 1990 Biomaterials 11509

Locardi B, Pazzaglia U E, Gabbi C and Profilo B 1993 Biomaterials 14437

Moreno E C, Kreask M and Zahradnik R T 1977 Carier Res. 11142

Narasaraju T S B 1972 Indian J. Chem. 10308

Okazaki M 1992 Biomaterials 13749

Osborn J F and Newesely H 1980 Biomaterials 1108

Partenfelder U, Engel A and Russel C 1993 J. Mater. Sci. Mater. Med. 4292

Roth R S and Negas T 1983 Phase diagrams for ceramists (Westerville; American Ceramic Society) p. 213

Roy D M and Linnehan S K 1974 Nature 247220 